\title{
CHARGE STATE EVOLUTION IN THE SOLAR WIND. II. PLASMA CHARGE STATE COMPOSITION IN THE INNER CORONA AND ACCELERATING FAST SOLAR WIND
}

\author{
E. Landi, J. R. Gruesbeck, S. T. Lepri, T. H. Zurbuchen, and L. A. Fisk \\ Department of Atmospheric, Oceanic and Space Sciences, University of Michigan, Ann Arbor, MI 48109, USA \\ Received 2012 July 13; accepted 2012 October 14; published 2012 November 20
}

\begin{abstract}
In the present work, we calculate the evolution of the charge state distribution within the fast solar wind. We use the temperature, density, and velocity profiles predicted by Cranmer et al. to calculate the ionization history of the most important heavy elements in the solar corona and solar wind: $\mathrm{C}, \mathrm{N}, \mathrm{O}, \mathrm{Ne}, \mathrm{Mg}, \mathrm{Si}, \mathrm{S}$, and Fe. The evolution of each charge state is calculated from the source region in the lower chromosphere to the final freeze-in point. We show that the solar wind velocity causes the plasma to experience significant departures from equilibrium at very low heights, well inside the field of view (within $0.6 R_{\text {sun }}$ from the solar limb) of nearly all the available remote-sensing instrumentation, significantly affecting observed spectral line intensities. We also study the evolution of charge state ratios with distance from the source region, and the temperature they indicate if ionization equilibrium is assumed. We find that virtually every charge state from every element freezes in at a different height, so that the definition of freeze-in height is ambiguous. We also find that calculated freeze-in temperatures indicated by charge state ratios from in situ measurements have little relation to the local coronal temperature of the wind source region, and stop evolving much earlier than their correspondent charge state ratio. We discuss the implication of our results on plasma diagnostics of coronal holes from spectroscopic measurements as well as on theoretical solar wind models relying on coronal temperatures.
\end{abstract}

Key words: solar wind - Sun: UV radiation

Online-only material: color figures

\section{INTRODUCTION}

The plasma charge state composition is of fundamental importance for any study of the solar atmosphere and solar wind. Close to the Sun, the charge state distribution determines the rate of emission of the plasma itself: thus, it shapes the continuum and line radiation of coronal plasmas which are observed by all high-resolution X-ray, EUV, and UV spectrometers and narrowband imagers currently deployed in space. The charge state distribution of solar plasmas is often assumed to be in equilibrium, except during sudden events of solar activity, such as the impulsive phase of flares and the acceleration phase of coronal mass ejections. As a consequence, plasma diagnostics, synthetic spectra calculation, radiative losses and energy budget estimates, as well as solar EUV irradiance evaluation are usually carried out under the assumption of ionization equilibrium.

Far from the Sun, the charge state composition of the solar wind is routinely measured by in situ instrumentation and it can be used to study the mechanisms of solar wind acceleration and evolution. Hundhausen et al. (1968) were the first to note that as the solar wind is accelerated outward from the Sun, the plasma density decreases quickly and causes the ionization and recombination rates to lose effectiveness at the same rate. Thus, the wind plasma ion composition is less and less responsive to changes in plasma temperature as it travels away from the Sun; eventually, the plasma "freezes in," that is, its charge state composition stops evolving no matter what dynamic and thermal evolution the plasma undergoes after the freeze-in point. This freeze-in point occurs within a few solar radii from the solar photosphere, so that the plasma properties measured by the current in situ instrumentation retain the memory of the physical processes the wind plasma has experienced very close to the Sun. Such properties can in principle be used to study the wind acceleration mechanisms in the very early stages, its trajectory, and to identify the wind source regions.
Several earlier studies found that carbon and oxygen ions freeze in within 1.3 $R_{\text {sun }}$ (Bürgi \& Geiss 1986; Ko et al. 1997; Chen et al. 2003; Landi et al. 2012a). For example, Ko et al. (1997) studied the evolution of the charge states of $\mathrm{C}, \mathrm{O}, \mathrm{Mg}$, $\mathrm{Si}$, and $\mathrm{Fe}$ in the fast solar wind and indicated that freeze-in occurs within two solar radii for all elements except Fe, which stopped evolving at around 4-5 $R_{\text {sun }}$. Also, Bürgi \& Geiss (1986) showed that significant departures from equilibrium occur well before the freeze-in point, at heights that can be reached by remote-sensing instrumentation observing the lines of each evolving charge state. Esser et al. (1998) carried out a detailed investigation of the evolution of the charge states of $\mathrm{C}, \mathrm{O}, \mathrm{Si}$, and $\mathrm{Fe}$ and found that the relatively fast velocity of minor ions observed by SOHO/UVCS (Kohl et al. 1997) induces significant departures from equilibrium; these results led Esser et al. (1998) to question the assumption of equilibrium commonly adopted in the interpretation of in situ measurements of charge state ratios. The results by Bürgi \& Geiss (1986), Ko et al. (1997), and Esser et al. (1998) were obtained using the ionization and recombination rate coefficients available at the time. Many new rate coefficients have been measured and calculated for many elements of interest in the last 35 years, so that these results need to be updated.

In this paper, we further develop the ideas in Bürgi \& Geiss (1986), Ko et al. (1997), and Esser et al. (1998) and investigate the evolution of the charge states in the fast solar wind below two solar radii with two goals. First, we will study in detail whether and up to what distance from the solar limb is the assumption of ionization equilibrium valid for all the ions that emit lines observed by the X-ray, EUV, and far UV spectrometers currently available. Second, we will determine the diagnostic potential of frozen-in ion charge states measured in situ to infer the electron temperature of the coronal plasma in the fast wind source regions. To do this, we will use the ionization and recombination rate coefficients in CHIANTI (Dere et al. 1997; 

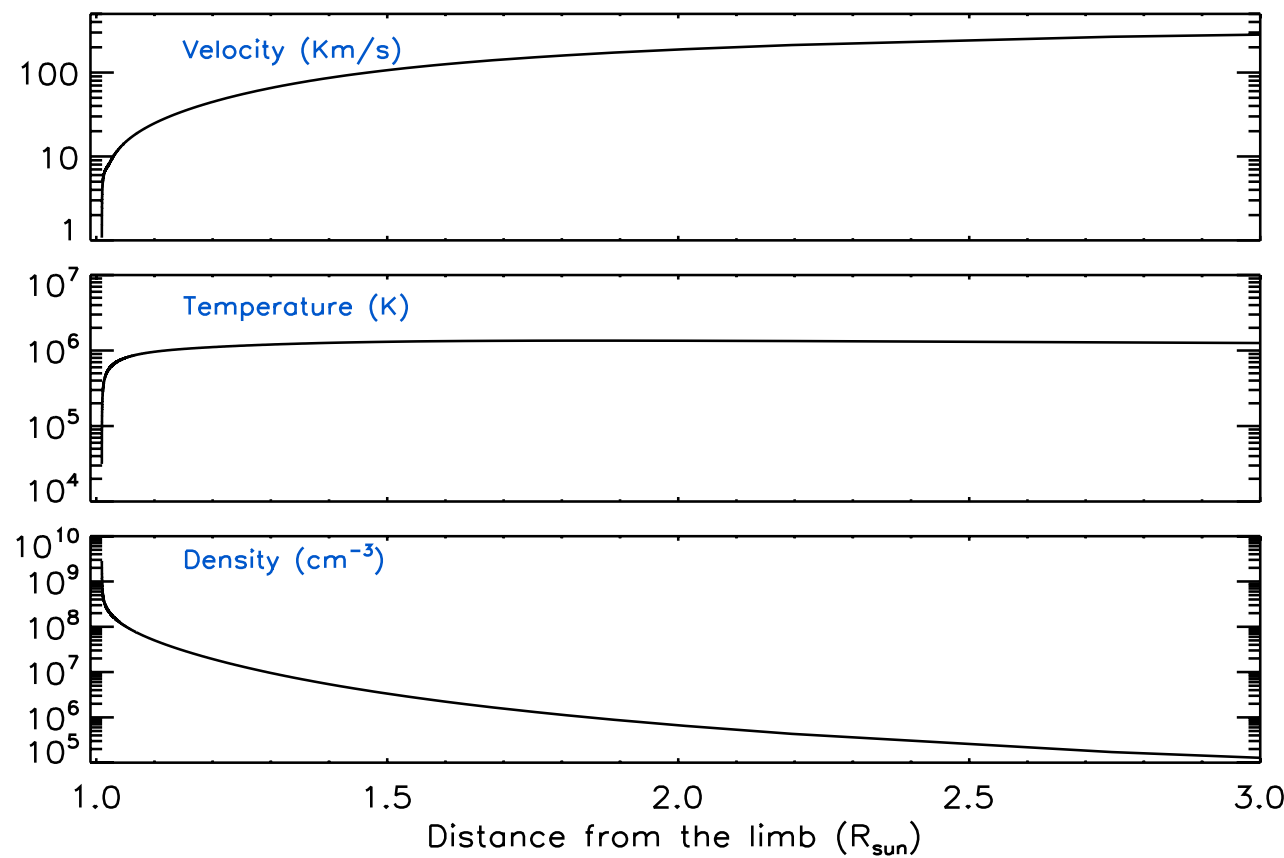

Figure 1. Wind temperature, electron density, and velocity as a function of distance for the coronal hole/fast wind model of Cranmer et al. (2007).

(A color version of this figure is available in the online journal.)

Landi et al. 2012b), which include much improved ionization and recombination rates published since 1998. The results of this work have been used to discuss the radiative losses in solar wind models by Landi et al. (2012d), and will also be used in following papers of this series to study the source region of the fast solar wind. They will also be tested against uncertainties in the ionization and recombination rate coefficients we used in another paper.

The calculation of the ion charge states is described in Section 2, the results are discussed in Section 3, and conclusions are drawn in Section 4.

\section{CHARGE STATES IN THE SOLAR WIND}

\subsection{Charge State Evolution Calculation}

To calculate the evolution of charge states in the rest frame of the expanding solar wind, it is necessary to solve, for each element, the equation

$$
\begin{aligned}
\frac{\partial y_{m}}{\partial t}= & n_{e}\left(y_{m-1} C_{m-1}\left(T_{e}\right)-y_{m}\left(C_{m}\left(T_{e}\right)+R_{m-1}\left(T_{e}\right)\right)\right. \\
& \left.+y_{m+1} R_{m}\left(T_{e}\right)\right) \\
\Sigma_{m} y_{m}= & 1,
\end{aligned}
$$

where $T_{e}$ is the electron temperature, $n_{e}$ is the electron density, $R_{i}$ and $C_{i}$ are the total recombination and ionization rate coefficients, and $y_{m}$ is the fraction of a given element in charge state $m$. The set of continuity equations for each element is solved numerically as a set of stiff ordinary differential equations using a fourth-order Runge-Kutta method. To solve this equation, we have used an adaptive step size, which is selected by forcing the maximum change in the abundance of any charge state to be smaller than $10 \%$ at any given step.

To solve this equation, two main ingredients are needed:

1. the electron temperature and density of the wind plasma as a function of time;

2. a complete database of ionization and recombination rate coefficients.
The first of these two ingredients can be given, for the problem of an expanding solar wind, if the electron density and temperature of the wind plasma is known along the entire trajectory of the wind itself. If the plasma parameters are assumed to be independent of time at any point along the wind trajectory, then the wind velocity determines how much time the wind plasma spends at any given density and temperature along its path. Wind plasma temperature, density, and velocity can be either taken from solar wind models or assumed by the user, and then adjusted empirically to reproduce both spectral line intensities close to the Sun and in situ charge state measurements, using the diagnostic technique outlined by Landi et al. (2012c).

Since in the present work we are interested in studying the evolution of the charge states in the fast solar wind, rather than determining the best temperature, velocity, and density profiles that fit a set of observations, we will use the temperature, density, and velocity profiles predicted by the Cranmer et al. (2007) wind model, hereafter C07. In this work, we focus on the fast solar wind and coronal holes, so we will use the coronal hole model provided by the authors. The velocity, density, and temperature curves of the model are shown in Figure 1. Esser et al. (1998) showed how charge state compositions are sensitive to the input density, temperature, and velocity profiles. C07 provides a very realistic representation of the density and temperature of coronal holes. Figure 2 shows a comparison between the temperature and density profiles from the $\mathrm{C} 07$ coronal hole model with measurements from Landi (2008) from a polar coronal hole. The agreement between predicted and observed (where available) values is very good.

The second ingredient necessary to the calculation requires a large database of ionization and recombination rate coefficients. Such databases have been developed in the past (e.g., Landini \& Monsignori Fossi 1990; Mazzotta et al. 1998; Dere 2007; Mattioli et al. 2007) and include data for direct ionization, excitation-autoionization, radiative recombination, and dielectronic recombination. Rates for all ions of all elements up to 

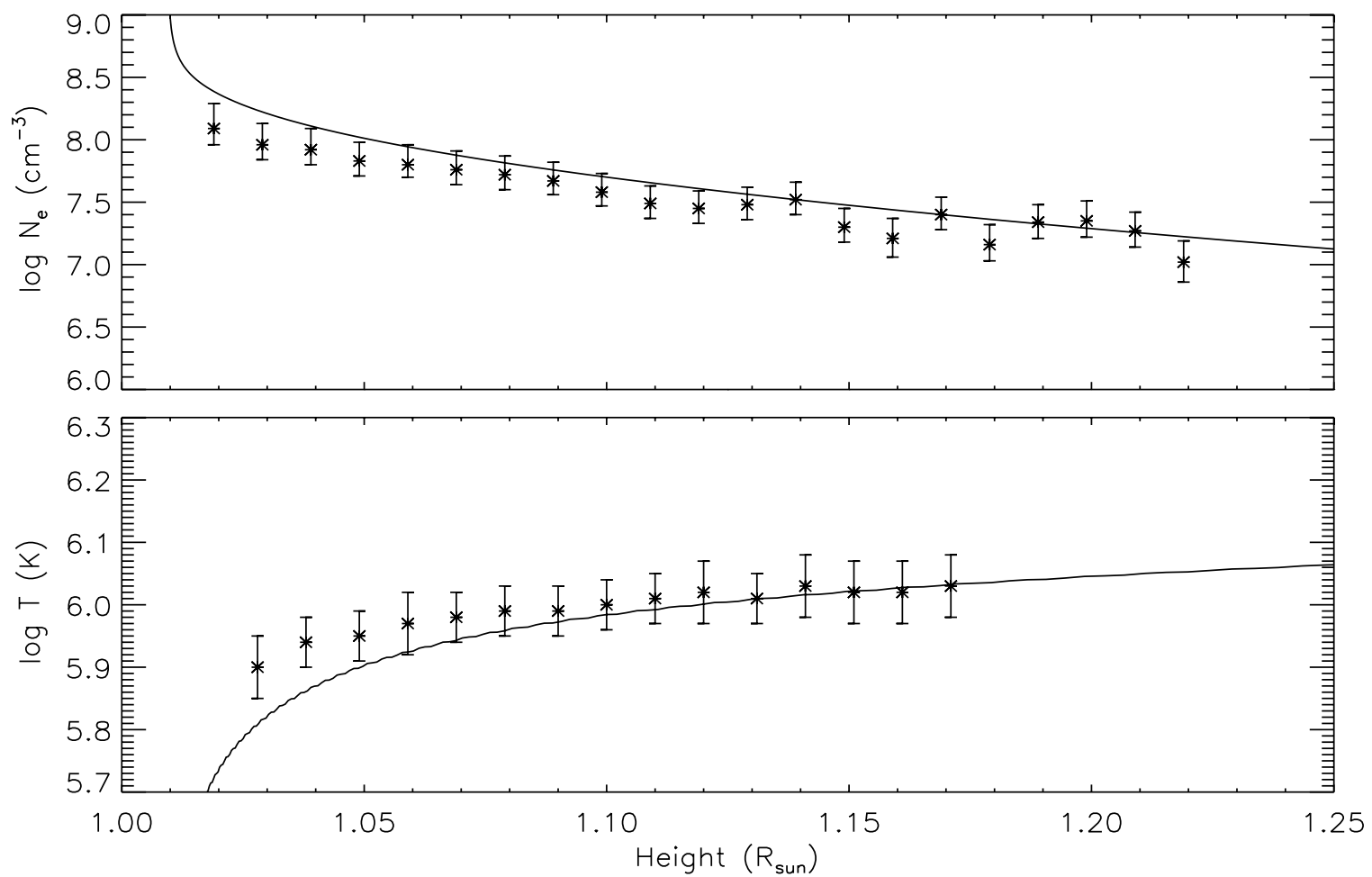

Figure 2. Comparison between temperature and density measurements of the temperature and density of a coronal hole (from Landi 2008) with predictions from the coronal hole/fast wind model of Cranmer et al. (2007).

zinc are included that can be readily used in Equation (1). In the present work, we will use the data in version 7 of the CHIANTI database, largely based on the Dere (2007) compilation for ionization rates, and on the review of recombination rates made in version 6 of CHIANTI (Dere et al. 2009). As a boundary condition, we assume that the plasma at $6600 \mathrm{~km}\left(0.01 R_{\text {sun }}\right)$ is in local thermodynamic equilibrium, so that the temperature and density at the boundary are $3.1 \times 10^{4} \mathrm{~K}$ and $2.8 \times 10^{9} \mathrm{~cm}^{-3}$, respectively, according to $\mathrm{C} 07$. Landi et al. (2012d) showed that the departures from equilibrium occur very early in the wind trajectory for the lightest elements such as $\mathrm{C}, \mathrm{N}$, and $\mathrm{O}$, so that the lower boundary condition of $200,000 \mathrm{~K}$ chosen by Esser et al. (1998) may be placed too high in the atmosphere for the lightest elements.

In our calculations, we implicitly assume that the electron velocity distribution is Maxwellian. Still, non-thermal electrons can be important, for example, Bürgi (1987) studied the effects of non-thermal electrons on the predicted charge state distribution, by using a $k$ distribution and found that they were significant, and could alter the temperature measured using charge state ratios. Also, $\mathrm{C} 07$ assumes that all ions flow at the same speed. Such an assumption might not be necessarily true in the real solar wind. However, even if the charge state evolution is very sensitive to variations in the wind velocity profile (Esser et al. 1998), the results obtained with the $\mathrm{C} 07$ nonetheless help illustrate the general behavior of all elements.

\subsection{Ionization and Recombination Trends}

Figures 3 and 4 show the timescales for total ionization from, and recombination to, all the ions of the most abundant heavy elements in the solar atmosphere, as a function of the number of electrons in each charge state, and as a function of the charge state. The timescales have been calculated for each ion as

$$
\tau=\frac{1}{n_{e} R}
$$

where $R$ is the total recombination (or ionization) rate coefficient for that ion, and $n_{e}$ has been assumed to be $n_{e}=10^{8} \mathrm{~cm}^{-3}$, a value typical of inner coronal hole regions. As the wind travels outward and the electron density decreases, the timescales in Figures 3 and 4 increase at the same rate. Also, the electron temperature of the plasma has been assumed to be $T=10^{6} \mathrm{~K}$, typical of the solar wind plasmas closest to the Sun, where the electron density is highest and ionization and recombination processes are most efficient. As temperature decreases with wind expansion in the outer corona, ionization timescales increase, while recombination rates decrease.

Figures 3 and 4 can be read in two ways. If one considers a fixed value of the number of electrons (following a so-called isoelectronic sequence), as in the left panels of both figures, both the ionization and recombination timescales increase smoothly as the atomic number increases. If the ionization and recombination timescales are considered for a given element (following the curve of a given color in the left panels), then the series of ionization and recombination timescales experienced by each element accelerating outward in the solar wind can be followed.

There are two main things that need to be noticed in Figures 3 and 4 . First, the recombination rates for all ions of all elements are within two order of magnitudes, while the variation of the ionization rates within each element can exceed six order of magnitudes. More importantly, the ionization rates increase dramatically for all of the elements as they enter the helium and neon isoelectronic sequences ( 2 and 10 electrons, respectively). Also, while the recombination timescales for the ions of the same element do not change much as the element ionizes 

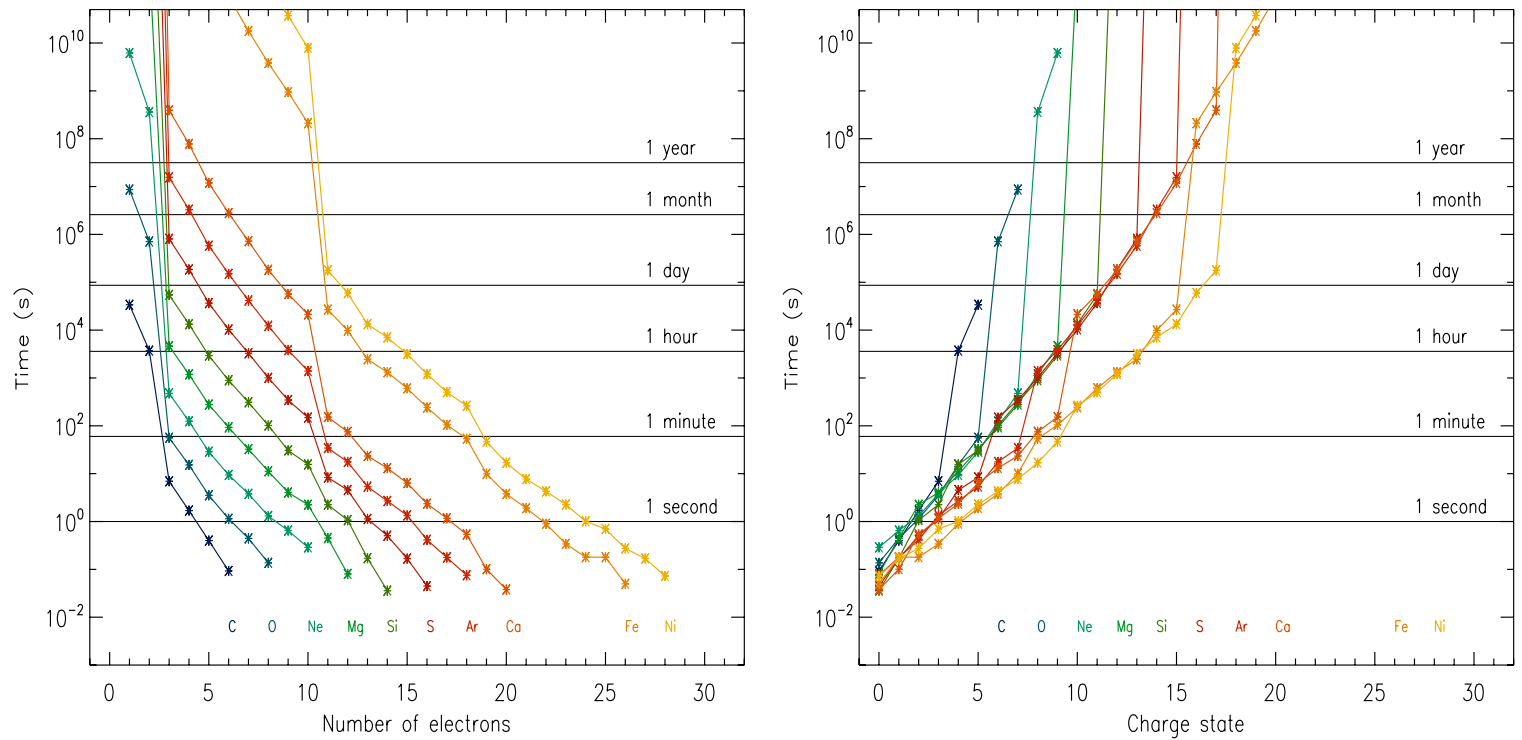

Figure 3. Total ionization timescales for all the ions of the most abundant heavy elements in the solar atmosphere, as a function of the number of electrons in each ion (left) and of the element's charge state (right). Data are taken from the CHIANTI database.

(A color version of this figure is available in the online journal.)
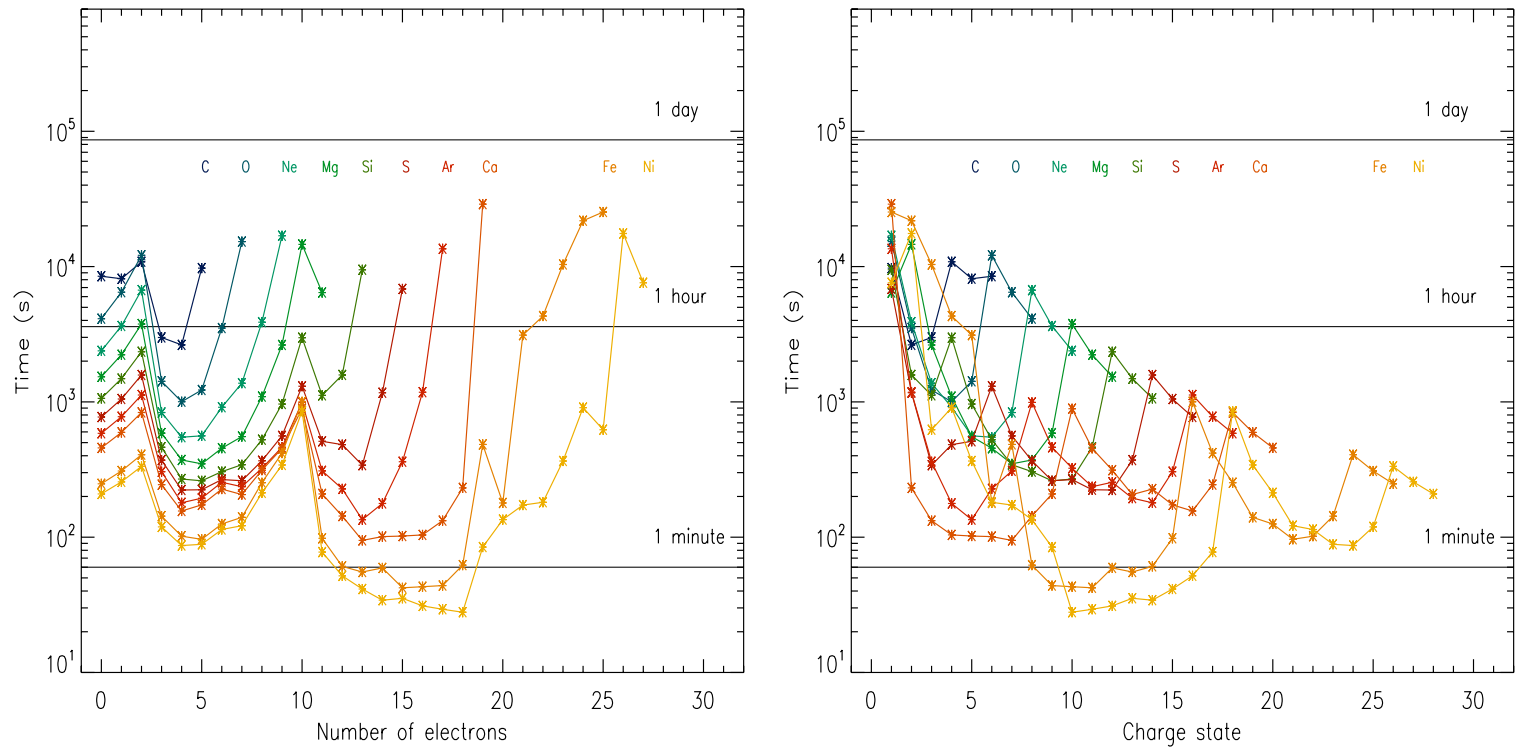

Figure 4. Total recombination timescales for all of the ions of the most abundant heavy elements in the solar atmosphere, as a function of the number of electrons in each ion (left) and of the element's charge state (right). Data are taken from the CHIANTI database.

(A color version of this figure is available in the online journal.)

from a very low charge state at the solar wind boundary in response to the rapidly rising temperature in the inner corona, its ionization timescales increase constantly. This means that as the charge state of the ion keeps increasing, each charge state encounters more and more difficulties in further ionizing, while its recombination timescale does not change much. Also, since each element is starting from a rather low charge state distribution at the wind boundary, it takes an increasingly longer time to ionize through every single charge state to reach the distribution typical of the solar corona even if the plasma density were constant rather than quickly decreasing. As a consequence, each element experiences some sort of "ionization inertia" that tends to leave it underionized unless it is given enough time to reach ionization equilibrium at the local temperature.

\section{RESULTS}

\subsection{Ion Charge State Evolution}

The predicted ion charge states as a function of distance from the limb are shown in Figures 5-8 for all of the most abundant heavy elements in the solar wind. Each of those figures shows two quantities: the absolute value of the charge state fraction $y_{m}$ for the most important species (such that $\Sigma_{m} y_{m}=1$ ), and the ratio between this value to the charge state fraction calculated under the assumption of equilibrium at the local temperature, to show to what extent the element departs from equilibrium. The horizontal lines in the charge state ratio panel indicate $\pm 25 \%$ from unity. Most of the ions shown in Figures 5-8 emit spectral lines that are routinely observed in coronal holes outside the disk by one or more of the available spectrometers on board Solar 

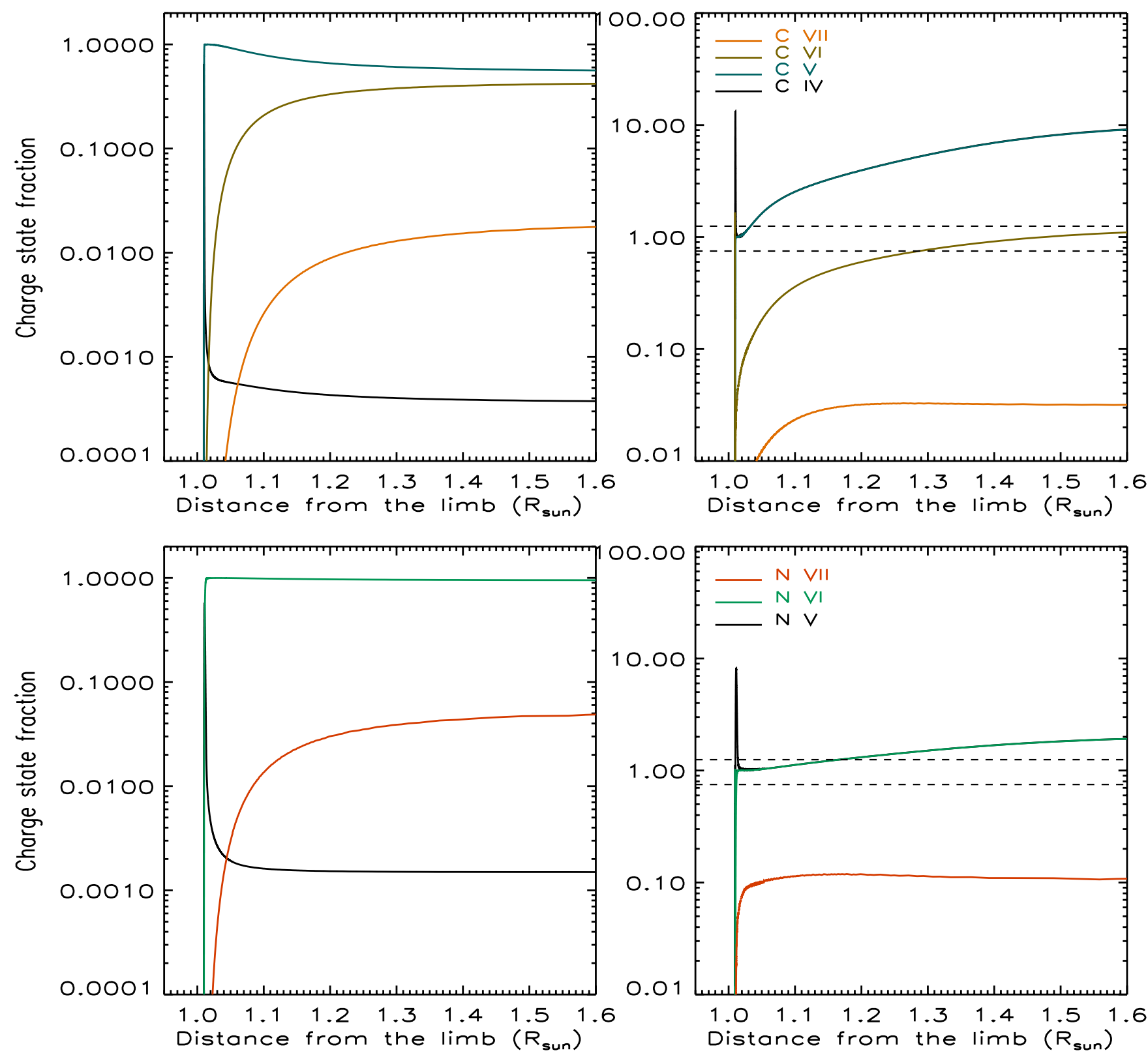

Figure 5. Charge state evolution for carbon (top) and nitrogen (bottom), as a function of distance from the solar limb (at $1 R_{\text {sun }}$ ). Left: absolute charge state fractional abundances. Right: ratio of wind charge state abundance to ionization equilibrium values calculated at the local electron temperature.

(A color version of this figure is available in the online journal.)

and Heliospheric Observatory ( $\mathrm{SOHO}$ ) or Hinode, so that they can in principle be used to study the evolution of the fast solar wind. We have shown the results up to $1.6 R_{\text {sun }}$ as this is the outermost limit of the field of view of the spectrometers on board $\mathrm{SOHO}$ and Hinode that observe the ions shown here (although $\mathrm{SOHO} / \mathrm{UVCS}$ reaches larger heights). This height also includes the field of view of all narrowband imagers currently available: SOHO/EIT, Transition Region and Coronal Explorer, STEREO/EUVI, Hinode/XRT, and SDO/AIA. The photosphere is set at $1.0 R_{\text {sun }}$.

Figures 5-8 tell several things. First, lighter elements stop evolving much earlier than the heavier elements; only with the exception of Fe, they are almost frozen in at around 1.5-1.6 $R_{\text {sun }}$. Even Fe, however, slows its evolution down considerably at that height. Second, most elements show significant departures from equilibrium. These departures occur well within the field of view of all available instruments.
Some systematics in the behavior of different elements can be identified. First, one of the most important results is that Fe charge states are for the most part within $20 \%$ of their equilibrium values for most of the field of view of the $\mathrm{SOHO}$ and Hinode spectrometers. The exceptions are Fe XIV, Xv: since the plasma temperature is still increasing at $1.6 R_{\mathrm{sun}}, \mathrm{Fe} \mathrm{XIV}, \mathrm{XV}$ show the effects of the ionization inertia. These two ions, however, provide very weak emission in coronal holes. The most important conclusion for $\mathrm{Fe}$ is that since the diagnostic studies carried out in the lower corona usually assume that the plasma is in ionization equilibrium, results obtained in coronal holes using Fe lines (such as the very bright ones observed by Hinode/EIS and $S O H O / C D S$ ) are confirmed even in the presence of an accelerating solar wind. The reason for this is that $\mathrm{Fe}$ ionization and recombination rates are fast enough to continue to be effective below $1.6 R_{\text {sun }}$, so that the plasma is able to adapt to local conditions rather efficiently. 

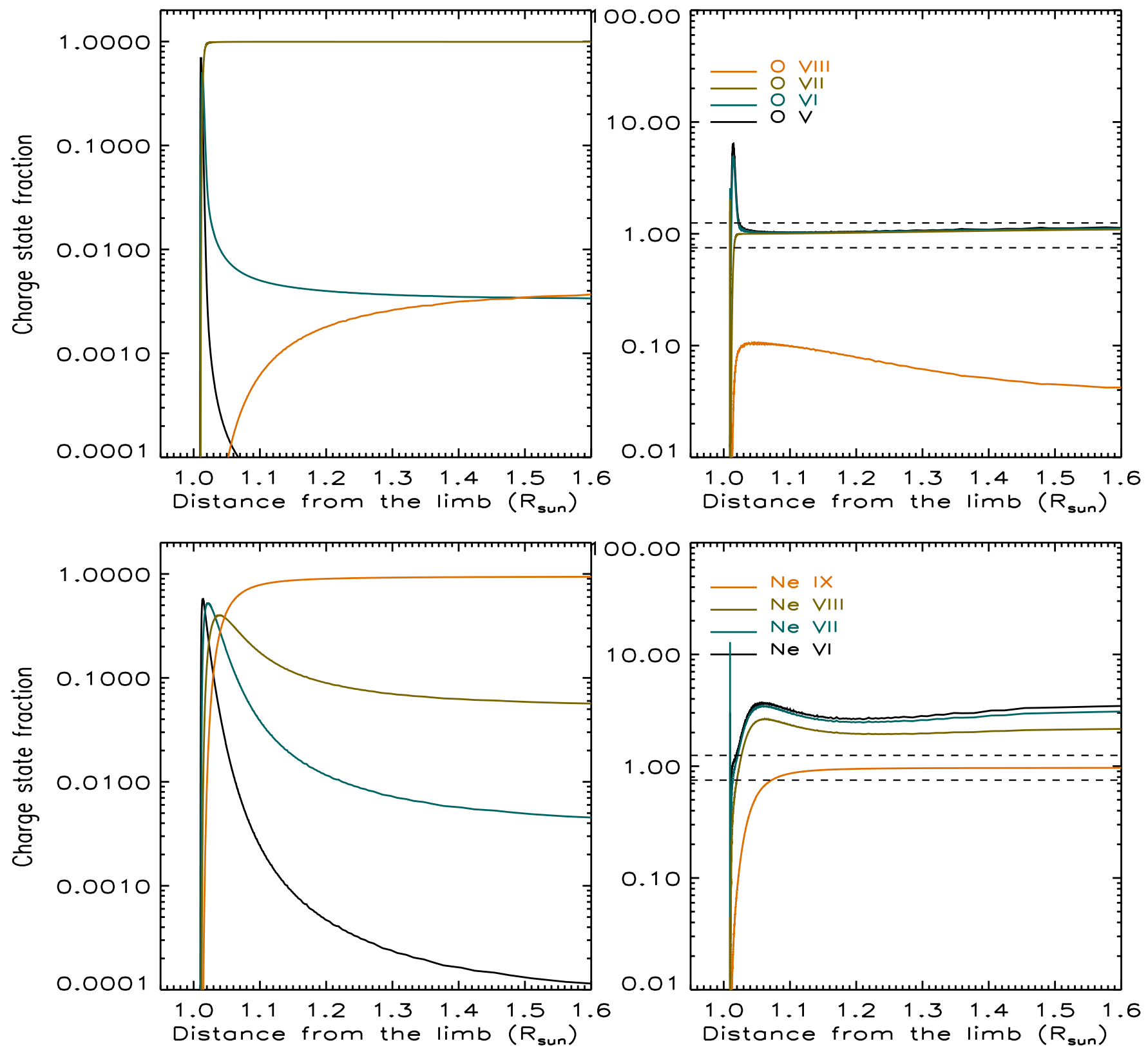

Figure 6. Charge state evolution for oxygen (top) and neon (bottom), as a function of distance from the solar limb (at $1 R_{\text {sun }}$ ). Left: absolute charge state fractional abundances. Right: ratio of wind charge state abundance to ionization equilibrium values calculated at the local electron temperature.

(A color version of this figure is available in the online journal.)

Second, the behavior of the lightest elements-carbon to neon-is dominated by the presence of the charge state with two electrons, belonging to the helium isoelectronic sequence. This charge state is quite peculiar, as elements with two electrons fill the innermost shell (the $1 s$ shell) in their atomic structure, so that it is very difficult to ionize them further, as shown by the large rise in the ionization timescales for these ions over those with more electrons (Figure 3). On the contrary, charge states with three or four electrons (lithium and beryllium isoelectronic sequences) have shorter ionization timescales. Since the boundary condition is set in the lower transition region, the plasma initial charge stage distribution is peaked toward very low charge states, so that the plasma ionizes as it travels outward. Given the timescales for the Be-like and Li-like ions, the plasma very rapidly reaches the He-like charge state, but then stops ionizing as the timescales to further ionize an
He-like ion are too slow. This means that almost all ions of carbon to neon reach the He-like charge state and either stop ionizing or, like carbon, ionize only with difficulty. This is the cause of the large departure from equilibrium (e.g., a factor of 10) of the abundance of the He-like stage of ionization in the lower transition region.

Furthermore, the large fraction (essentially unity) of He-like ions, coupled with the relatively fast recombination time for an He-like ion into an Li-like ion, ensures that Li-like species receive a steady supply of recombining ions at little expense to the total fraction of the He-like ion; at the same time, the fast ionization time for the $\mathrm{Li}$-like ion ensures that the Li-like ion ionizes as if it was under equilibrium. This balance ensures that the He-like and Li-like charge states of nitrogen and oxygen fake near-equilibrium conditions to large altitudes even if some of their ion fractions have stopped evolving almost immediately 

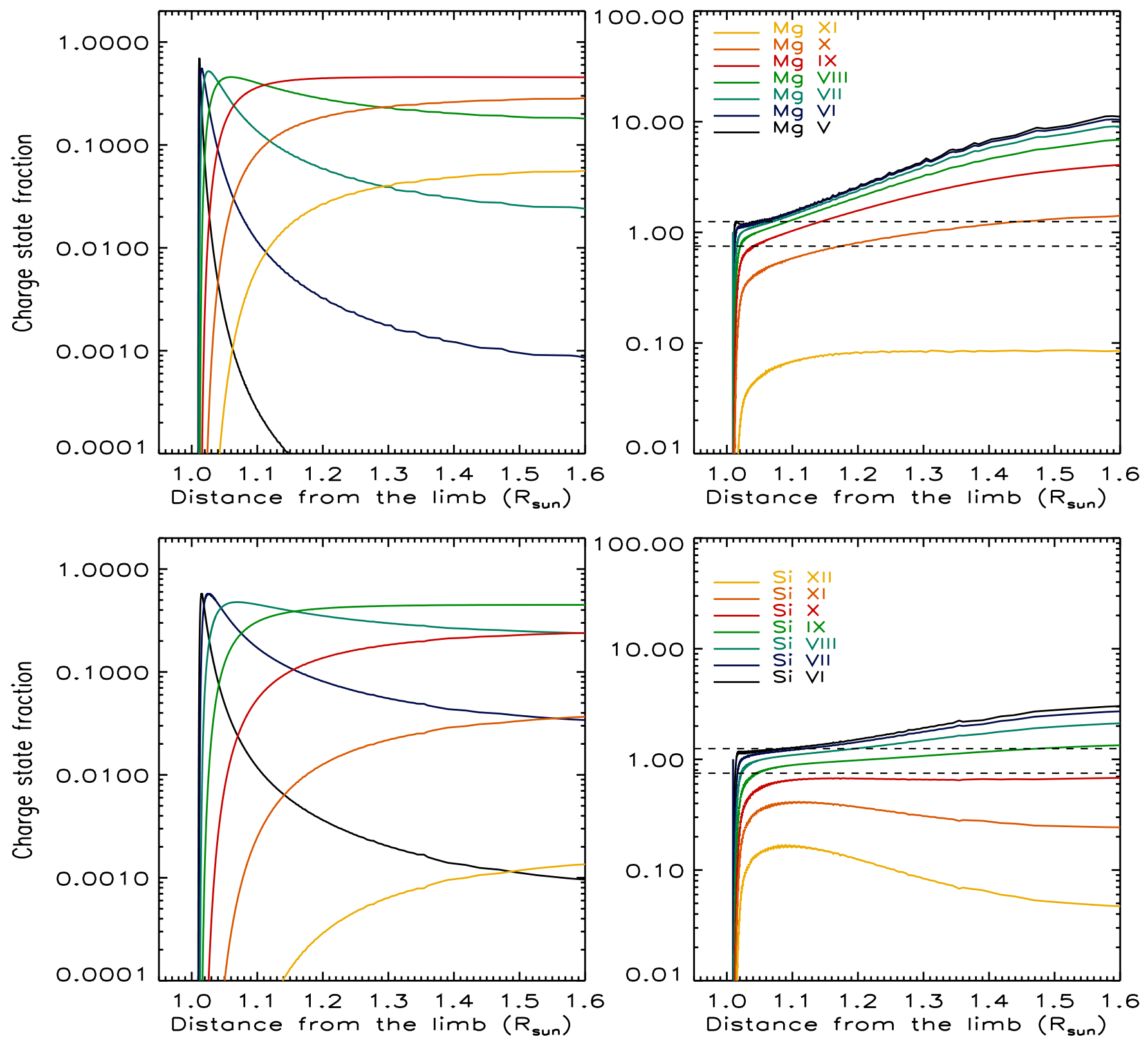

Figure 7. Charge state evolution for magnesium (top) and silicon (bottom), as a function of distance from the solar limb (at $1 R_{\text {sun }}$ ). Left: absolute charge state fractional abundances. Right: ratio of wind charge state abundance to ionization equilibrium values calculated at the local electron temperature.

(A color version of this figure is available in the online journal.)

upon entering the solar corona. Before this fake equilibrium is established, the Li-like and Be-like charge states keep ionizing at a pace that does not allow them to reach equilibrium at the local plasma temperature, so they become overabundant giving rise to the sharp spike in the transition region (Figures 5 and 6). This behavior has been noted by Landi et al. (2012a) for oxygen, and is very important to understand the evolution this element, which is one of the best observed elements in in situ measurements and is routinely used to infer the temperature of source regions of the solar wind. For carbon, the plasma temperature is too large to keep the He-like ion $(\mathrm{C} \mathrm{v})$ from further ionizing, so that the $\mathrm{H}$-like charge state stage slowly forms. For neon, the initial charge state distribution at the boundary is very far from the Helike stage, so that this element reaches the He-like charge state stage relatively late. We call the large enhancement of the Belike and $\mathrm{Li}$-like charge states in the lower transition region the "cold effect" because it affects ions that are mostly formed in the (relatively cold) transition region under equilibrium conditions.

The behavior of elements from magnesium to sulfur is qualitatively different from that of carbon and oxygen. Since the boundary conditions require that the charge state distribution be centered on very low ionization stages, these ions are ionizing as the solar wind accelerates outward. The main drivers of their behavior are that (1) their ionization and recombination timescales (as shown in Figures 3 and 4) are more or less in the same range, (2) they do not have the time (or a high enough temperature) to reach the He-like stage, and (3) there is no other "catalyst" charge state like the He-like one to prevent them from further ionizing. As the wind moves outward, the plasma quickly loses electrons and reaches the first coronal charge states that emit observable spectral lines very soon when the plasma distribution is still close to equilibrium. Thus, the lower 

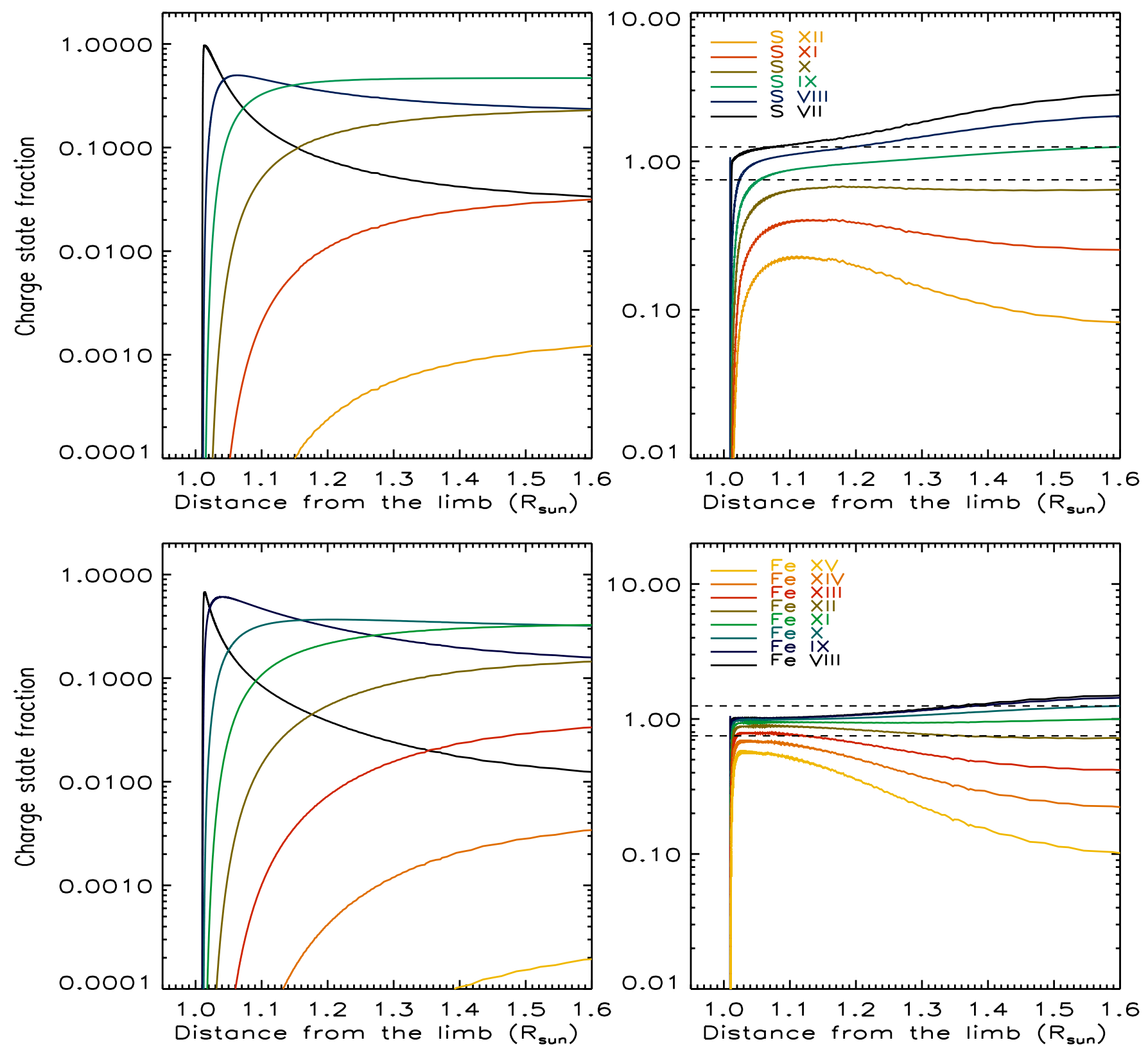

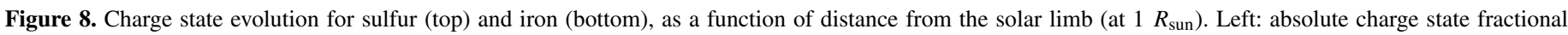
abundances. Right: ratio of wind charge state abundance to ionization equilibrium values calculated at the local electron temperature.

(A color version of this figure is available in the online journal.)

coronal charge states behave close to equilibrium. However, these elements do not have time enough to reach higher charge states, which thus have smaller abundances than the equilibrium values. This is the effect of the ionization inertia. Examples of such stages are $\mathrm{MgX}$, Si X,XI, S X-XII. However, ionization inertia also affects the lower coronal charge states, although at larger altitudes. In fact, as the plasma moves forward, the density decreases (Figure 1) so that ionization becomes less effective and charge states do not ionize fast enough to adapt to the hotter local conditions. As a consequence, the ions that were initially close to equilibrium become overabundant. This happens for Mg V-IX, Si VI-VIII, and S VII-VIII. The abundance of some of these ions have nonetheless become sufficiently low to make their lines unobservable, but some of them (Si vIII, Mg VIII-IX) can still be observed for larger distances so that these non-equilibrium effects are expected to affect the observed line intensities. We call this systematic behavior of coronal ions the "delay effect".

\subsection{Interpretation of In Situ Charge States}

In situ instrumentation allows the determination of the charge state distribution of the local solar wind. Charge state abundances have been used in two different ways. First, the (normalized) distribution of charge states can be directly compared to predictions from a model, to investigate the freeze-in process of the wind plasma. Second, the abundance ratios of individual charge states of the same element can be compared to theoretical estimates (made under the assumption of ionization equilibrium) of the ratio as a function of temperature: the comparison allows us to determine the temperature at which the wind plasma freezes in. These two quantities have routinely been used in the 

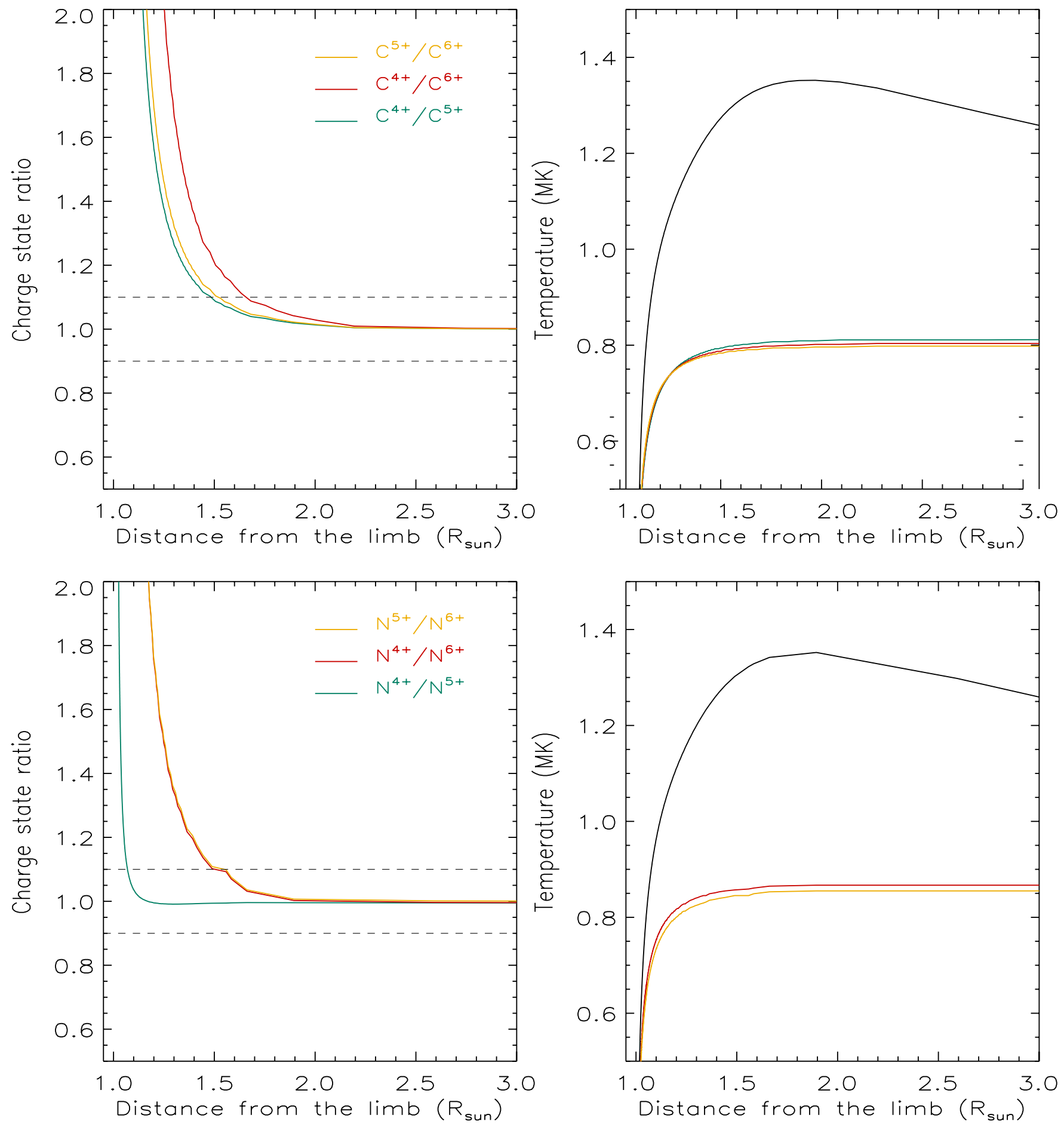

Figure 9. Evolution of charge state ratios and temperatures for carbon (top) and nitrogen (bottom) as a function of distance from the solar limb (at $1 R_{\text {sun }}$ ). Left: charge state ratios. Right: plasma temperature obtained comparing the charge state ratios in the left panel with estimates calculated as a function of temperature under the assumption of local ionization equilibrium. The black line indicates the temperature in the Cranmer et al. (2007) model. The $N^{4+} / N^{5+}$ charge state ratio was not monotonically decreasing with temperature so unique temperature values could not be derived.

(A color version of this figure is available in the online journal.)

literature to determine (1) at which height each element freezes in and (2) the temperature of the solar wind source region.

Figures 9-12 report the evolution of the charge state ratios and of the temperature diagnostics obtained from them, for $\mathrm{C}, \mathrm{N}, \mathrm{O}, \mathrm{Ne}, \mathrm{Mg}, \mathrm{Si}, \mathrm{S}$, and $\mathrm{Fe}$ ions. In each figure, the left panel shows the ratios between the most important charge states, which can be identified in in situ measurements and also provide observable spectral lines in the inner corona. These ratios have been normalized to their frozen-in values, and the horizontal dashed lines mark where these ratios are within $10 \%$ of the frozen-in value. Figures 9-12 show several things. First, within each element, each charge state ratio approaches the final frozenin value at a different height. We take the $10 \%$ mark as an indication of where a charge state ratio is close to freeze-in and define $R_{10}$ as the corresponding height. The differences 

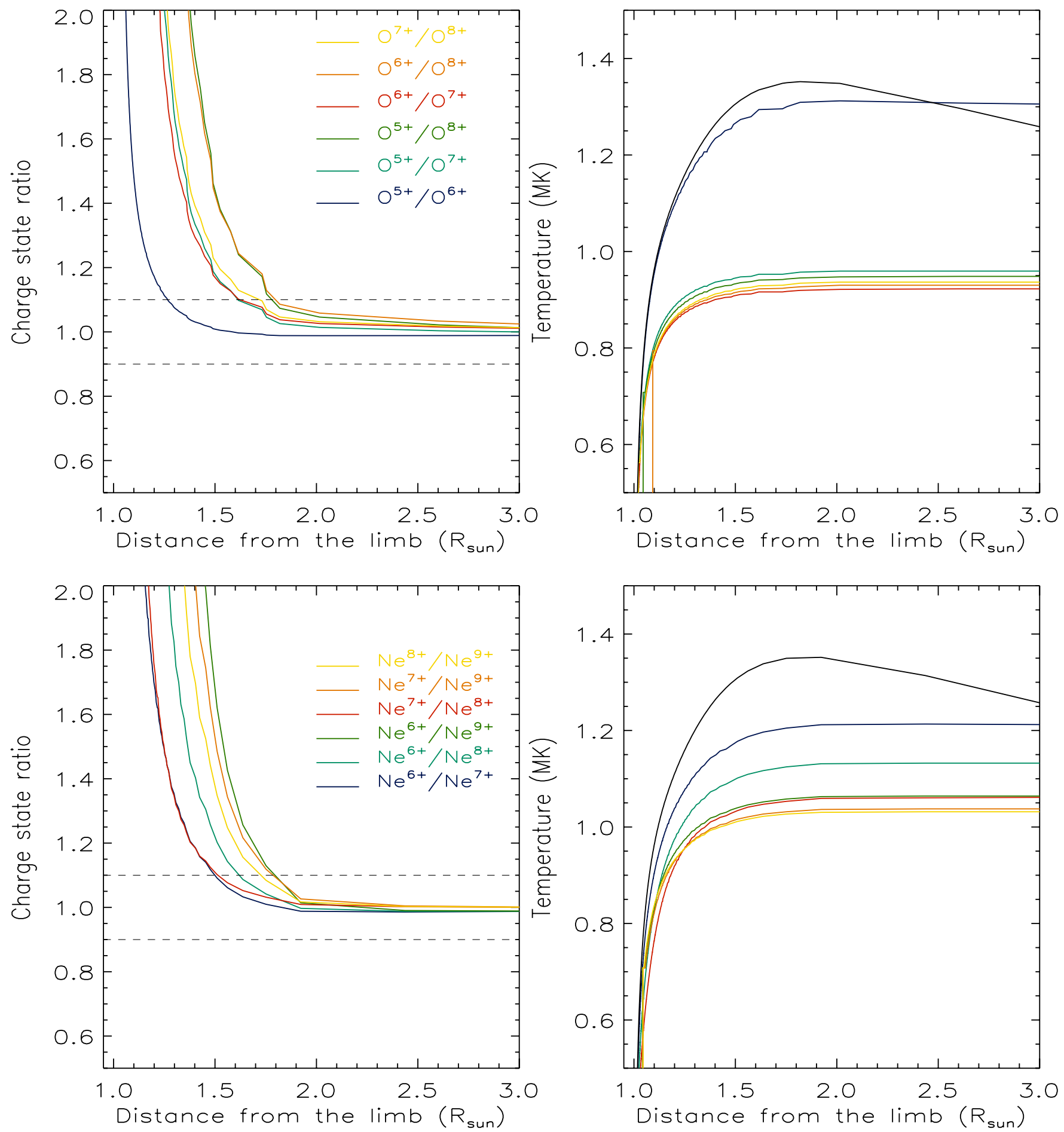

Figure 10. Evolution of charge state ratios and temperatures for oxygen (top) and neon (bottom) as a function of distance from the solar limb (at $1 R_{\text {sun }}$ ). Left: charge state ratios. Right: plasma temperature obtained comparing the charge state ratios in the left panel with estimates calculated as a function of temperature under the assumption of local ionization equilibrium. The black line indicates the temperature in the Cranmer et al. (2007) model.

(A color version of this figure is available in the online journal.)

in $R_{10}$ values are most evident in $\mathrm{N}$ and $\mathrm{O}$, where they can reach $0.5 R_{\text {sun }}$ : this behavior is a consequence of the presence of the He-like ionization stage in the element's distribution. All other elements also have differences in $R_{10}$ that can easily reach $\Delta R_{10} \simeq 0.2-0.3 R_{\text {sun }}$, the smallest being those for carbon ions, as noted by Landi et al. (2012a). Another thing to note is that even if individual charge state ratios stop evolving below $1.5 R_{\text {sun }}$, the element itself is still evolving; the final freeze-in occurs between 1.5 and $2.5 R_{\text {sun }}$. Last, Fe charge state ratios do not stop evolving and eventually react in response to the decreasing temperature up to $3.0 R_{\text {sun }}$.

The right panels in Figures 9-12 show the temperature calculated at each point along the wind trajectory using the calculated charge state ratios and the CHIANTI ion abundances under the ionization equilibrium assumption. The first thing to note is that for each element, the measured temperature "freezes in" much earlier than the charge state ratio itself. This is due to the fact that under the equilibrium assumption, the 

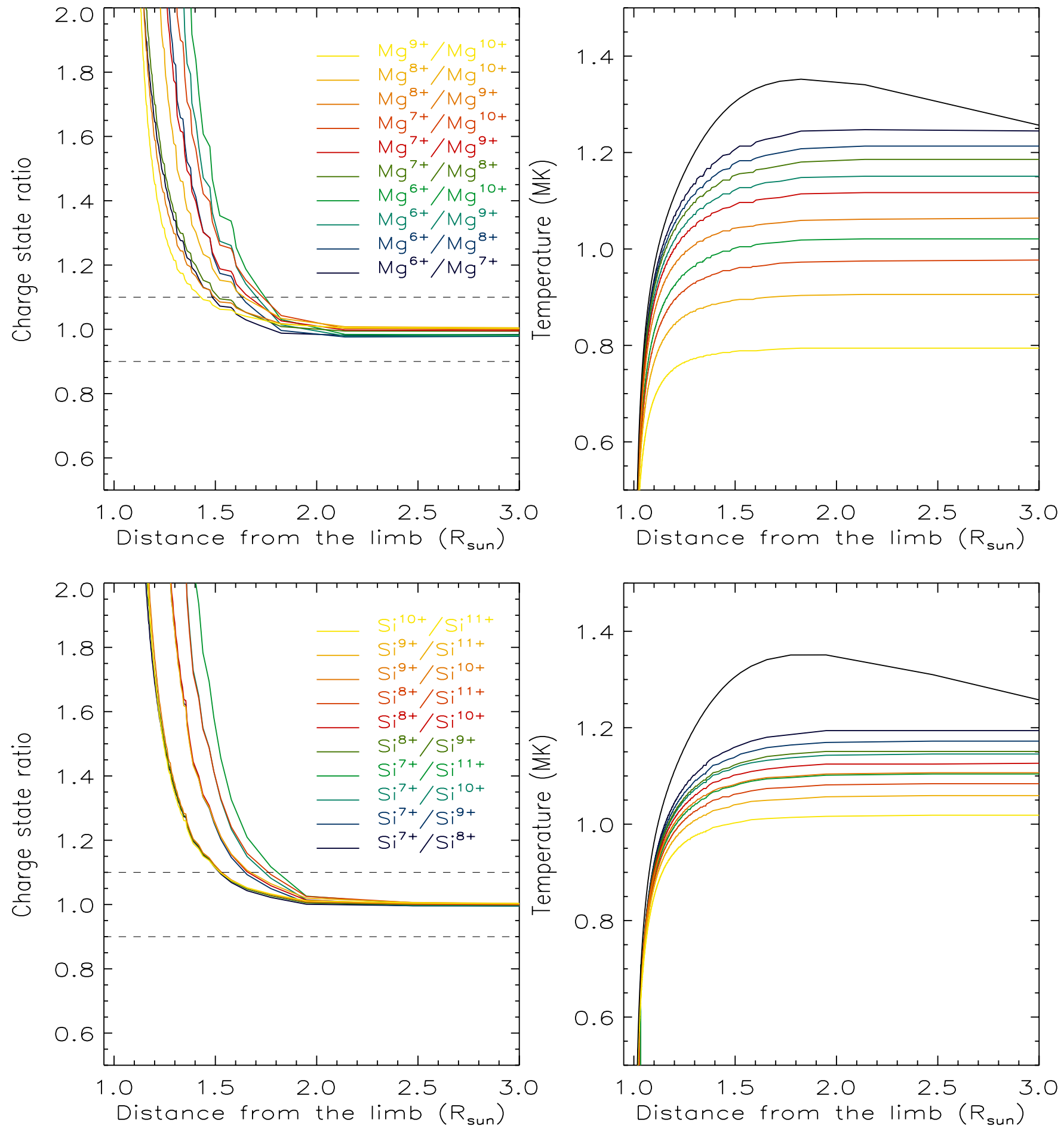

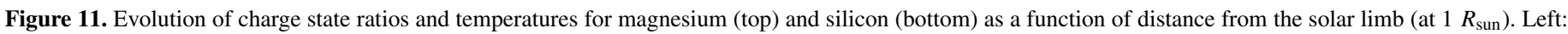

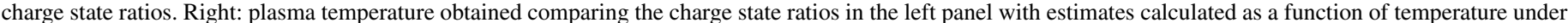
the assumption of local ionization equilibrium. The black line indicates the temperature in the Cranmer et al. (2007) model.

(A color version of this figure is available in the online journal.)

relative abundance of two different charge states is very sensitive to the electron temperature, so that even small variations of the latter may cause charge state ratios to change by one order of magnitude. Thus, as charge states approach the final frozen-in value, variations in their ratios are too small to provide significant temperature variations. As a consequence, the temperatures determined using in situ measurements of charge states correspond to the values reached before true freezein occurs.
When the temperature values provided by each element are inspected, two main things become apparent. First, within each element, different charge state ratios can provide very different temperature values; second, these temperature values are much different from the local plasma temperature (indicated in Figures $9-12$ by the black line, from the C07). These two results are a strong warning toward the use of in situ measurements to determine the plasma temperature of the wind source regions. For example, while $\mathrm{C}$ and $\mathrm{N}$ charge state 

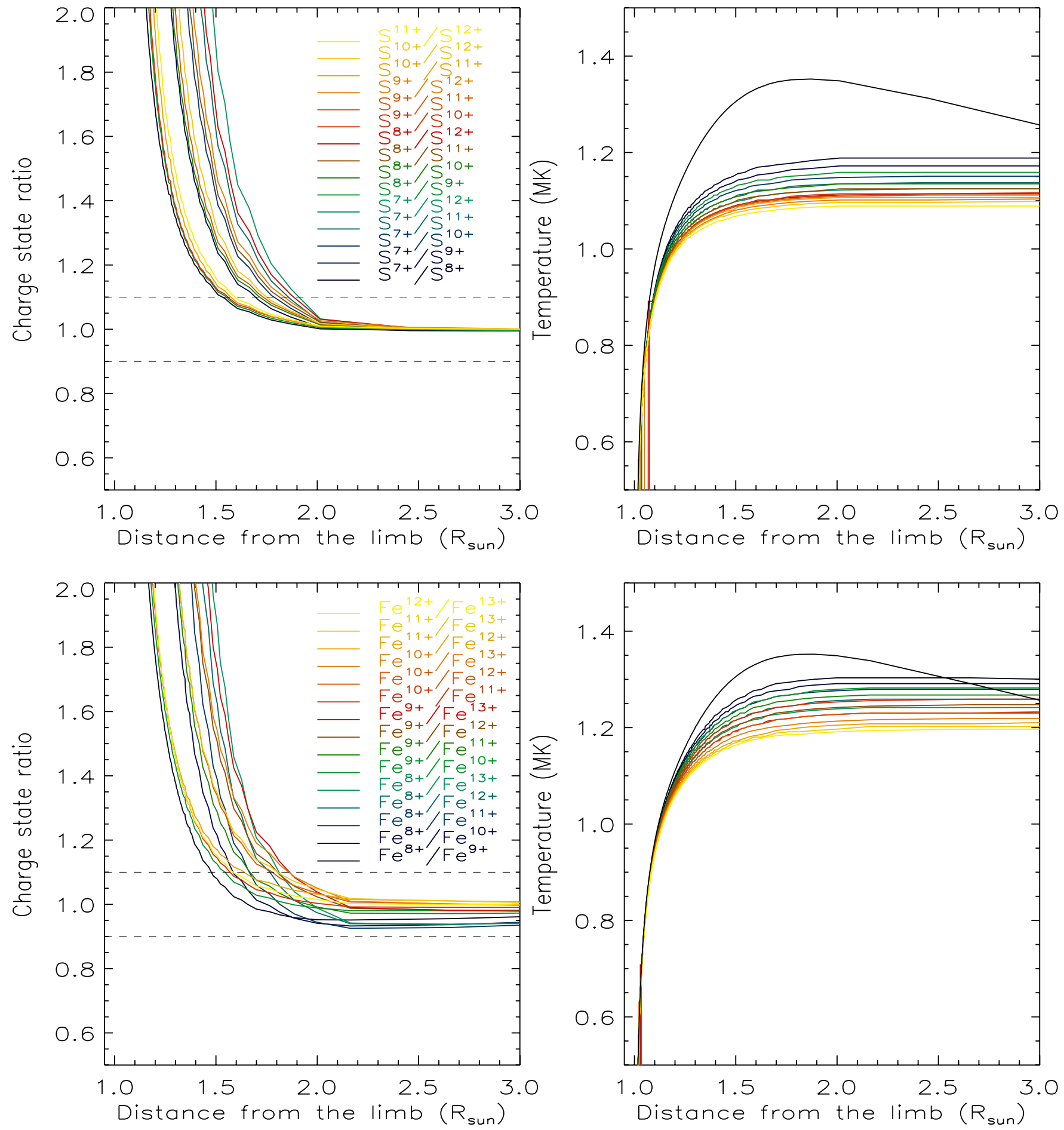

Figure 12. Evolution of charge state ratios and temperatures for sulfur (top) and iron (bottom) as a function of distance from the solar limb (at $1 R_{\text {sun }}$ ). Left: charge state ratios. Right: plasma temperature obtained comparing the charge state ratios in the left panel with estimates calculated as a function of temperature under the assumption of local ionization equilibrium. The black line indicates the temperature in the Cranmer et al. (2007) model.

(A color version of this figure is available in the online journal.)

ratios seem to provide self-consistent temperatures (different by a factor of 1.5 from the values at the height where their temperatures freeze in), the values provided by $\mathrm{O}, \mathrm{Ne}$, and $\mathrm{Mg}$ can change by a factor of 1.5 depending on which ratio is used. Most importantly, all of these temperature values have no relation either to the temperature of the source region (far below, in the chromosphere), or to the temperature of the freeze-in point, or to the temperature of the location where the temperature provided by the charge state ratio freezes in.

\section{DISCUSSION}

The results obtained in the present work have consequences both for the analysis of remote sensing observations, and of in situ measurements of charge state composition.

Figures 5-8 clearly show that almost all ions that emit bright lines observed in coronal holes are out of local equilibrium conditions. The abundance of most ions is larger than their equilibrium value, while the highest stages of ionization of 
heavier elements may be underabundant, because of the delay effect. Departures from equilibrium occur at heights that are well within the field of view of available spectrometers observing the solar disk (that is, below $1.5 R_{\text {sun }}$ ), so that their effects on line intensities are expected to be significant. Also, ions emitting key bright lines in the transition region, such as C IV, $\mathrm{N} \mathrm{v}, \mathrm{O}$ v, vi, and $\mathrm{Ne} \mathrm{vI}$, are out of equilibrium by large amounts at very low heights. As a consequence, the commonly adopted assumption of ionization equilibrium needs to be taken with caution. The effects of these departures on radiative losses in the solar atmosphere have been discussed by Landi et al. (2012d), who showed that the cold effect can provide significant changes to the radiative losses and energy equation of the fast solar wind in the transition region.

There are a few notable exceptions to these departures. First, $\mathrm{OV}$ and $\mathrm{O}$ vi fake ionization equilibrium in the corona even if oxygen as a whole is not in ionization equilibrium. This fake equilibrium lasts up to $4.0 R_{\text {sun }}$. This is important, as O vi emits one of the strongest doublets in the solar UV coronal spectrum at $1031.9 \AA$ and $1037.6 \AA$, which has been extensively studied in off-disk coronal spectra both from SUMER (up to $\approx 1.5 R_{\text {sun }}$ ) and UVCS (up to $\approx 4.0 R_{\text {sun }}$ ): results based on absolute line intensities and the equilibrium assumption for $\mathrm{O}$ VI are not affected by departures from equilibrium. The second notable exception is Fe VIII-XII, whose ion abundances also are within $20 \%-25 \%$ of their equilibrium values. This is also important, as these ions emit very strong lines in the EUV spectrum between $170 \AA$ and $200 \AA$ which are routinely observed and used for plasma diagnostics in coronal holes by the EIS spectrometer on board Hinode up to $\approx 1.5 R_{\text {sun }}$. These ions provide a core group of lines that can be relatively safely used to measure the plasma physical properties without worrying about nonequilibrium effects in their ion abundances. Among the available spectrometers, SUMER is the most affected by wind-induced non-equilibrium effects as it includes a large number of lines from almost all the ions shown in Figures 5-8 (e.g., Curdt et al. 2001, 2004). This provides a unique opportunity to use SUMER observations of lines from such ions to quantify such departures and empirically determine the temperature, density, and velocity profiles of the solar wind that best reproduce observed spectral line intensities using the diagnostic technique outlined by Landi et al. (2012c).

The results of the present investigation also affect measurements obtained in the heliospheric portion of the solar wind trajectory, from in situ instruments. In fact, one of the most important wind property provided by such instruments is the charge state distribution, which is routinely used to obtain freeze-in temperatures from charge state ratios. These temperatures are in turn used in some theoretical models as proxies of the temperature of the solar corona. For example, Gloeckler et al. (2003), Fisk (2003), and Schwadron \& McComas (2003) interpreted or developed solar wind scaling laws using relatively simple energy considerations. They were able to tie both the fast and solar wind properties to common types of physical processes occurring in the solar corona. Schwadron \& McComas (2003) assumed that the energy injection of solar wind particles into the source regions is the same (and roughly fixed) for both fast and slow solar winds, and that part of this energy is lost by radiation in the lower atmosphere; the amount of energy radiated away depends on the competition between energy conduction from the hot solar corona to the cooler (and more efficiently radiative) transition region, and energy advection due to the outward particle flux. These two competing processes are characterized by a single temperature $T_{\max }$, defined as the maximum temperature near or below one scale height (where most of the radiative losses take place). The Schwadron \& McComas (2003) model results in solar wind scaling laws that apply to all types of solar wind and in principle can explain the main physical properties of both types of wind. The basic principles of the model are rooted in the energy equation, so that the application of the Schwadron \& McComas (2003) model requires the specification of $T_{\max }$ to determine the final amount of energy lost through radiation. Schwadron \& McComas (2003) utilized the freezingin temperature of carbon from von Steiger et al. (2000) from the SWICS instrument on board Ulysses (Gloeckler et al. 1992), as carbon freezes in first, and thus the temperatures obtained from its charge state ratios were thought to be the best proxies for the coronal temperatures. However, Figure 9 clearly shows that the temperatures obtained from any carbon charge state ratios, while self-consistent, grossly underestimate the plasma peak temperature and are not representative of the temperature of the inner corona as predicted by $\mathrm{C} 07$. As their values are qualitatively and quantitatively disconnected from the real coronal temperature, they lead to incorrect estimates of the wind heat conduction and radiative losses and thus to an incorrect application of the solar wind scaling laws.

There is one last remark that needs to be made on the results of the present work. The evolution of the charge states, charge state ratios, and temperature of the fast solar wind that we have discussed depend critically on the plasma velocity, electron temperature, and electron density used for the calculation, as shown by Esser et al. (1998). Thus, they are dependent on the particular theoretical model used for the calculation, in this case C07. Other wind models can easily lead to different scenarios. However, the basic processes at play in the evolution of the solar wind charge states are rooted not only in the wind parameters, but also in the ionization and recombination rate coefficients that characterize its evolution. Regardless of any particular deviation of the temperature or the density history experienced by the wind plasma from $\mathrm{C} 07$ predictions, consecutive charge states of the same element will experience a slower ionization time while their recombination rates will change less, as shown by Figures 3 and 4 . Thus, a solar wind starting from the chromosphere is likely to experience both the delay effect and the cold effect, and its final charge state distribution will be delayed relative to the actual temperature of the corona. In fact, Zurbuchen et al. (2012) calculated the charge state evolution of the solar wind using an array of solar wind models (Hansteen \& Leer 1995; Leer et al. 1998; C07; van der Holst et al. 2010) and found that their final frozen-in charge states shared a similar behavior. They also found that such values were significantly lower than in situ distributions from carbon, oxygen, silicon, and iron, indicating that the delay effect introduced by each of these models leads to significant underestimates of the solar wind charge state composition.

In this work, we were interested in highlighting some general trends in the behavior of the solar wind, both very close to the Sun and beyond the freeze-in point. A more detailed and quantitative comparison of the charge state composition and EUV/UV line intensities predicted using C07 as well as other models is currently being pursued and will be the topic of a future publication.

The work of E.L. is supported by the NNX11AC20G and NNX10AQ58G, and grant AGS-1154443 from NSF. J.R.G. is supported by the GRSP program through grant NNX10AM41H. 
T.H.Z. and S.T.L. are supported by NASA through contract NNX08AI11G and grants NNX07AB99G and NNX10AQ61G. The authors warmly thank Dr. S.R. Cranmer for kindly providing the theoretical temperature, density, and velocity profiles used in this work. We also thank the anonymous referee for comments that helped us significantly improve and clarify this paper.

\section{REFERENCES}

Bürgi, A. 1987, J. Geophys. Res., 92, 1057

Bürgi, A., \& Geiss, J. 1986, Sol. Phys., 103, 347

Chen, Y., Esser, R., \& Hu, Y. 2003, ApJ, 582, 467

Cranmer, S. R., van Ballegooijen, A. A., \& Edgar, R. J. 2007, ApJS, 171, 520

Curdt, W., Brekke, P., Feldman, U., et al. 2001, A\&A, 375, 591

Curdt, W., Landi, E., \& Feldman, U. 2004, A\&A, 427, 1045

Dere, K. P. 2007, A\&A, 466, 771

Dere, K. P., Landi, E., Mason, H. E., Monsignori Fossi, B. C., \& Young, P. R. 1997, A\&AS, 125, 149

Dere, K. P., Landi, E., Young, P. R., et al. 2009, A\&A, 498, 915

Esser, R., Edgar, R. J., \& Brickhouse, N. S. 1998, ApJ, 498, 448

Fisk, L. A. 2003, J. Geophys. Res., 108, 1157

Gloeckler, G., Geiss, J., Balsiger, H., et al. 1992, A\&AS, 92, 267

Gloeckler, G., Zurbuchen, T. H., \& Geiss, J. 2003, J. Geophys. Res., 108, 1158

Hansteen, V. H., \& Leer, E. 1995, J. Geophys. Res., 100, 21577
Hundhausen, A. J., Gilbert, H. E., \& Bame, S. J. 1968, ApJ, 152, 3

Ko, Y.-K., Fisk, L. A., Geiss, J., Gloeckler, G., \& Guhathakurta, M. 1997, Sol, Phys., 171, 345

Kohl, J. L., Noci, G., Antonucci, E., et al. 1997, Sol. Phys., 175, 613

Landi, E. 2008, ApJ, 685, 1270

Landi, E., Alexander, R. L., Gruesbeck, J. R., et al. 2012a, ApJ, 744, 100

Landi, E., Del Zanna, G., Young, P. R., Dere, K. P., \& Mason, H. E. 2012b, ApJ, 744,99

Landi, E., Gruesbeck, J. R., Lepri, S. T., \& Zurbuchen, T. H. 2012c, ApJ, 750,159

Landi, E., Gruesbeck, J. R., Lepri, S. T., \& Zurbuchen, T. H. 2012d, ApJ, 758, L21

Landini, M., \& Monsignori Fossi, B. C. 1990, A\&AS, 82, 229

Leer, E., Hansteen, V. H., \& Holzer, E. 1998, in Proc. ESO Workshop, Cyclical Variability in Stellar Winds, ed. L. Kaper \& A. W. Fullerton (Berlin: Springer), 263

Mattioli, M., Mazzitelli, G., Finkenthal, M., et al. 2007, J. Phys. B: At. Mol. Opt. Phys., 40, 3569

Mazzotta, P., Mazzitelli, G., Colafrancesco, S., \& Vittorio, N. 1998, A\&AS, 133,403

Schwadron, N. A., \& McComas, D. J. 2003, ApJ, 599, 1395

van der Holst, B., Manchester, W. B., Frazin, R. A., et al. 2010, ApJ, 725,1373

von Steiger, R., Schwadron, N. A., Hefti, S., et al. 2000, J. Geophys. Res., 105,27217

Zurbuchen, T. H., von Steiger, R., Gruesbeck, J. R., et al. 2012, Space Sci. Rev., 172,41 